Article

\title{
Effect of Meniscus Damping Ratio on Drop-on-Demand Electrohydrodynamic Jetting
}

\author{
Samuel Haedong Kim ${ }^{1}$, Heuiseok Kang ${ }^{2}$, Kyungtae Kang ${ }^{2}$, Sang Ho Lee ${ }^{2}$, Kwan Hyun Cho ${ }^{2}$ \\ and Jun Young Hwang ${ }^{2, *}$ \\ 1 Woodruff School of Mechanical Engineering, Georgia Institute of Technology, Atlanta, GA 30332, USA; \\ samuel.h.kim@gatech.edu \\ 2 Korea Institute of Industrial Technology, Cheonan 31056, Korea; hskang@kitech.re.kr (H.K.); \\ ktkang@kitech.re.kr (K.K.); sholee7@kitech.re.kr (S.H.L.); khcho@kitech.re.kr (K.H.C.) \\ * Correspondence: jyhwang@kitech.re.kr; Tel.: +82-10-5285-7074
}

Received: 15 December 2017; Accepted: 23 January 2018; Published: 24 January 2018

\begin{abstract}
Drop-on-demand (DOD) electrohydrodynamic (EHD) jet printing uses a nozzle and pulsated electric fields to eject small ink droplets of functional material to the appointed spot of a substrate at the appointed time, which offers solutions of high resolution patterning for fabrication of printed electronics, bioengineering, and display. Because the EHD jet connects fine drops to yield a fine pattern, it is essential to realize high throughput by generating drops quickly and reliably. In this study, the characteristics of jetting frequency were experimentally investigated as a function of nozzle dimensions by measuring response of jetting frequency to pulsating frequency which is varying from $1 \mathrm{~Hz}$ to $2000 \mathrm{~Hz}$. The results showed that, even when the nozzle diameter is the same, the other dimensions of the nozzle significantly change the response of jetting to high pulsating frequency. Using a linear damping model describing hydrodynamic motion of ink inside the nozzle, the different behavior of the jetting frequency was explained via the different damping ratio of the oscillating ink: contrary to an underdamped system, an overdamped system supports a jetting frequency higher than the natural frequency.
\end{abstract}

Keywords: electrohydrodynamic jetting; drop-on-demand; jetting frequency; damping ratio; natural frequency

\section{Introduction}

Direct printing of functional electronic materials has attracted considerable interest. This method combines simple additive processing with low-cost materials and have a potential to drastically lower the cost of electronics fabrication, especially compared to that for conventional silicon processing [1]. Inkjet printing, which represents a highly established direct printing method, has been demonstrated to be capable of printing all materials required for electronics, display, optics, bioengineering, and other areas. However, there is a critical challenge of inkjet printing in that practically feasible resolution is as low as $20-30 \mu \mathrm{m}$. On the other hand, electro-hydro-dynamic jet (EHD-jet) printing provides high-resolution patterning $(<10 \mu \mathrm{m})$, which means that this process has potential for application in nano-systems such as NEMS and biotechnology [2]. EHD-jet printing is a technique that uses electric fields between the nozzle and an opposing conducting substrate to make the functional electronic material flow from a nozzle via electro-hydro-dynamics. There have been studies to understand the fundamental mechanism of EHD-jet printing; however, the physics of EHD-jet printing and the parameters that affect the printing are not yet clearly understood, and these parameters will be significant for high resolution, uniform, and reproducible printing.

Meniscus deformation was investigated by changing the imposed voltage such that when the bias voltage increases, not only the meniscus height increases, but also the chance of jetting of smaller drops 
with lower pulse voltage also increases [3-5]. By changing the applied voltage and flow rate, jet type was also studied such that, as the flow rate and voltage increase, the jet-mode changes from dripping, to pulsating, to cone-jet, to tilted-jet, and to multi-jet [6,7]. Also, a number of studies investigated the effects of amplitude and frequency of electric voltage pulse on the jetting and printing characteristics for drop-on-demand (DOD) printing systems with various inks and nozzles [8-14]. In those previous studies, examined jetting frequency was relatively low (typically less than $100 \mathrm{~Hz}$ ) because an EHD system has little means to stabilize the motion of the meniscus right after the detachment of the drop. This is because filling the nozzle with ink takes some time to form a stabilized drop for repeatable jetting. Understanding the movements of ink inside the nozzle is, therefore, crucial to achieve high speed jetting with small drops in advanced manufacturing.

Stachewiez et al. [15] proposed a useful model to describe the electro-hydro-dynamic movements of ink in the nozzle as an oscillatory system to explain the jetting stability with the electric pulse frequency, which introduces the natural frequency and damping ratio of the system as a function of ink property and nozzle geometry. They clearly showed that, in their underdamped jetting system with $50 \mu \mathrm{m}$ diameter nozzle, the maximum jetting frequency is limited by the natural frequency. Interestingly, if the nozzle diameter decreases sufficiently, the damping ratio can increase to make this an overdamped system. Compared with the underdamped system, the overdamped system might be stable at the higher jetting frequency. However, direct evidence for the higher stability of the overdamped system has not been given yet.

Considering this background, for EHD jetting of Ag nanoparticle ink, this study investigates the effect of the damping ratio on the stability against pulsating frequency. EHD nozzles having various damping ratios were tested by adopting different nozzle geometries; the relation between jetting frequency and pulsating frequency was measured. The results for the overdamped system were compared with those for the underdamped system. The effect of pulsating amplitude on jetting stability was also investigated for both overdamped and underdamped systems.

\section{Experimental Setup}

Figure 1a is a schematic of the experimental setup for the EHD printing system. In this study, we used silver ink (DGP 40LT-15C), which contains 30 35\% silver particles dissolved in Triethylene Glycol Monoethyl Ether (TGME), from ANP Co., Ltd. (Sejong, Korea) Specific resistivity of the ink is $11 \sim 12 \mu \Omega-\mathrm{cm}$, such that the ink can easily be used to produce electronics. Density, surface tension, and viscosity of ink are $1450 \mathrm{~kg} / \mathrm{m}^{3}, 35 \mathrm{mN} / \mathrm{m}$, and $15 \mathrm{mPa}-\mathrm{s}$, respectively. Three glass nozzles having different dimensions were tested, as shown in Table 1.

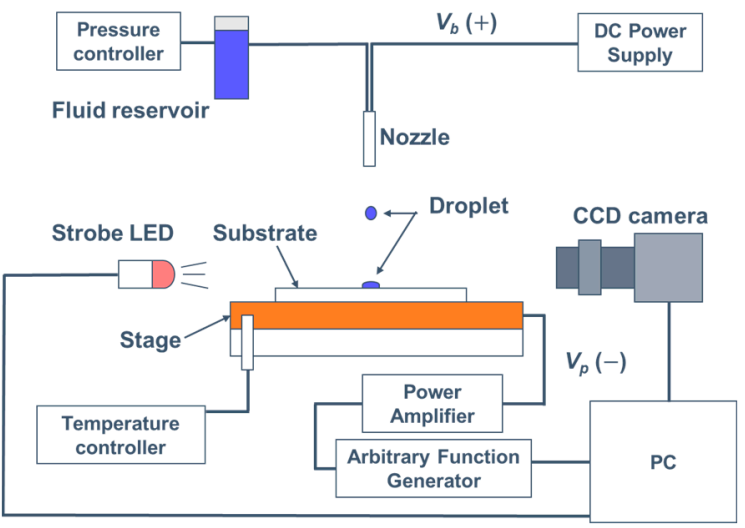

(a)

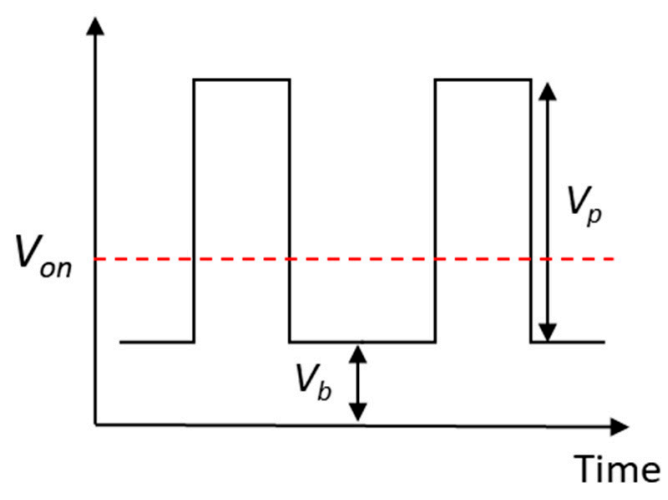

(b)

Figure 1. (a) Schematic of electro-hydro-dynamic (EHD) drop generation system; (b) Waveform applied to EHD system: bias voltage $\left(V_{b}\right)$ is applied to the nozzle, lower than the onset voltage $\left(V_{o n}\right)$, and pulsed voltage $\left(V_{p}\right)$ is applied to the stage. 
Table 1. Parameters of the experimental set-up.

\begin{tabular}{llll}
\hline \multirow{2}{*}{ Parameter } & Nozzle \# & \\
\cline { 2 - 4 } & A & B & C \\
\hline Approximate ink column height, $H(\mathrm{~m})$ & 0.2 & & \\
Distance between nozzle and substrate, $h(\mu \mathrm{m})$ & 100 & & \\
Inner diameter of nozzle, $d_{n}(\mu \mathrm{m})$ & 30 & & 15 \\
Radius of the capillary far above the nozzle, $b(\mu \mathrm{m})$ & 375 & 120 & 60 \\
Total length of the thinnest part, $l(\mathrm{~mm})$ & 3.0 & 1.2 & 1.2 \\
Bias voltage, $V_{b}(\mathrm{~V})$ & 650 & 620 & 450 \\
\hline
\end{tabular}

Bias voltage is applied to the nozzle by a high voltage DC power supply (ConverTech, Gyeonggi, Korea, SHV 300R). To minimize the droplet size and facilitate generation of drops with low pulse voltage, bias voltage was applied at $90 \%$ of onset voltage, $V_{\text {on }}$, at which point EHD-jet starts to form, as shown in Figure $1 \mathrm{~b}[4,5]$. The following equation estimates the value of $V_{\text {on }}[16,17]$

$$
V_{o n} \sim d_{n} \ln \left(\frac{8 h}{d_{n}}\right) \sqrt{\frac{\left(\frac{4 \gamma}{d_{n}}-\Delta P\right)}{8 \varepsilon}}
$$

where $d_{n}$ is the inner diameter of the nozzle, $h$ is the distance between the nozzle and the substrate, and $\gamma$ is the surface tension of the ink; according to the Young-Laplace equation, capillary pressure in a tube is $4 \gamma / d_{n}, \Delta P$ is the hydrostatic pressure, and $\varepsilon$ is the permittivity of air, which was assumed to be $8.859 \times 10^{-12} \mathrm{~F} / \mathrm{m}$ at room temperature [18]. The value of $V_{\text {on }}$ calculated using Equation (1) is compared with the experimental results using nozzle A, as shown in Figure 2; the results agree to each other.

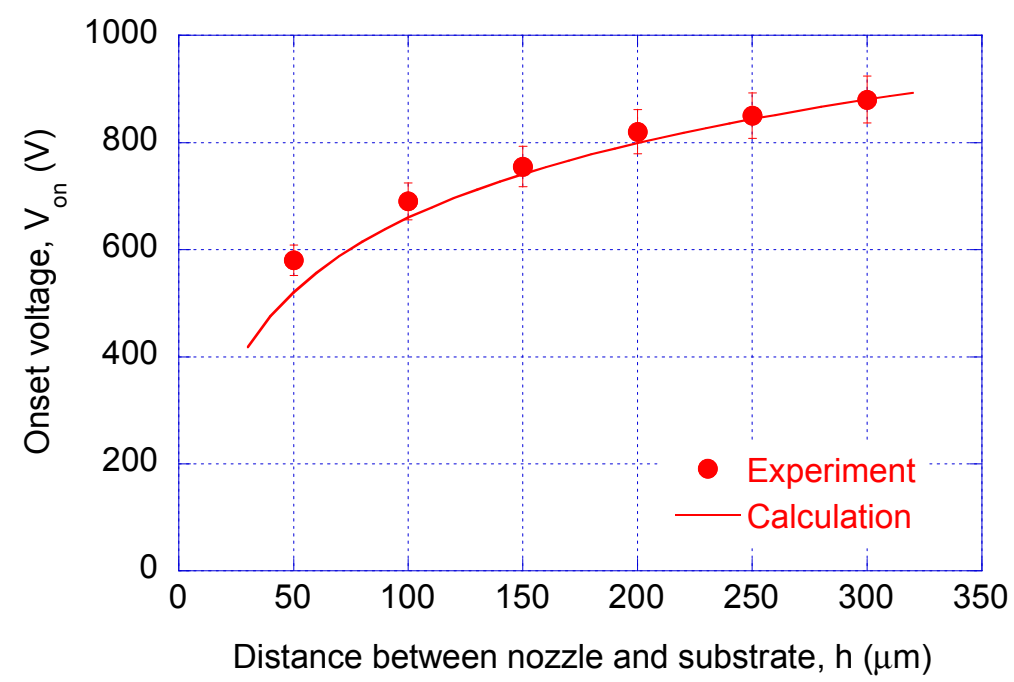

Figure 2. Onset voltage, $V_{o n}$, as a function of distance between nozzle and substrate. Calculated data is based on Equation (1), and experimental data were obtained using Nozzle A $\left(d_{n}=30 \mu \mathrm{m}\right)$.

A complex-waveform driver (MicroFab INC., Plano, TX, USA, JetDrive III) is used to generate a rectangular step-function signal, which is sent to the power amplifier (TREK INC., Lockport, NY, USA, TREK 2220) for $200 \mathrm{~V} / \mathrm{V}$ amplification to make the voltage pulse, $V_{p}$ having a duration of $500 \mu \mathrm{s}$, as shown in Figure 1b. In this experiment, pulsation frequency was varied from 1 to $2000 \mathrm{~Hz}$ while moving speed of the stage was set to the pulsation frequency multiplied by $40 \mu \mathrm{m}$. Therefore, the ratio of the pulsation frequency to the jetting frequency of the ink could be obtained by measuring the printed drop spacing divided by $40 \mu \mathrm{m}$. 


\section{Linear Damping Approximation}

The electrohydrodynamic movements of the ink in the capillary can be described by the equation of linear damping system [19]

$$
m \ddot{x}+c \dot{x}+k x=0
$$

where $m$ is the mass, $c$ is the damping coefficient, $k$ is the spring constant, and $x$ is the ink displacement inside the capillary nozzle. The components of Equation (2), the natural oscillation frequency $f_{c}$, and the damping ratio $\Gamma$, of the EHD-jet system were modeled by the following equations [19]

$$
\begin{aligned}
& m=\frac{\pi}{4} d_{n}^{2} \rho H \\
& c=8 \pi v l \\
& k=\frac{\pi \gamma d_{n}{ }^{4}}{2\left(\frac{d_{n}^{2}}{4}+h_{m}^{2}\right)^{2}}\left(1-\frac{2 h_{m}^{2}}{\frac{d_{n}^{2}}{4}+h_{m}^{2}}\right)+\rho g \pi \frac{d_{n}^{4}}{16 b^{2}} \\
& f_{c}=\frac{1}{2 \pi} \sqrt{\frac{k}{m}} \\
& \Gamma=\frac{c}{2 \sqrt{m k}}
\end{aligned}
$$

where $H$ is the approximate ink column height, $v$ is the viscosity of the ink, $l$ is the total length of the thinnest part, $h_{m}$ is the approximate meniscus height, which is approximated by $20 \%$ of the inner diameter of nozzle [15], $b$ is the radius of the capillary far above the nozzle, as illustrated in Figure 3. All values are shown in Table 1 . Table 2 shows the calculated natural frequency and the damping ratios of the four different nozzles. Regarding the dimensions of the nozzle and the property of the ink, the damping ratios of nozzle $A$ and $C$ are higher than 1 , and that of nozzle $B$ is smaller than 1; these values correspond to overdamped and underdamped systems, respectively.

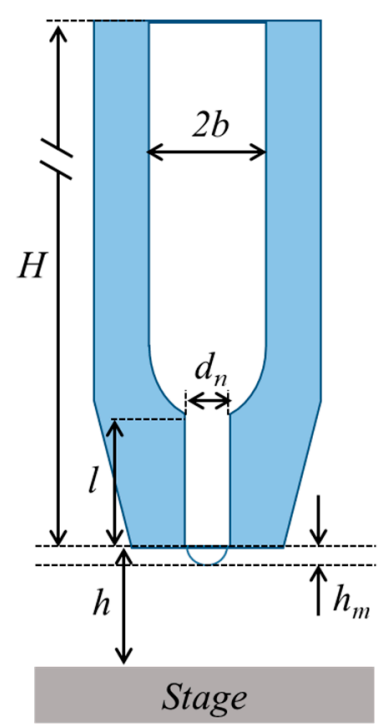

Figure 3. Schematics of nozzle: $\rho=$ density of ink, $\gamma=$ surface tension of ink, $v=$ viscosity of ink, $H=$ approximate ink column height from ink reservoir to free end of nozzle, $b=$ radius of capillary far above nozzle, $h=$ distance between nozzle and substrate, $h_{m}=$ meniscus height, $d_{n}=$ inner diameter of nozzle, and $l=$ total length of thinnest part of nozzle. 
Table 2. Calculated natural frequency and damping ratio of three different nozzles.

\begin{tabular}{|c|c|c|c|}
\hline \multirow{2}{*}{ Parameter } & \multicolumn{3}{|c|}{ Nozzle \# } \\
\hline & A & B & $\mathrm{C}$ \\
\hline Natural frequency inside the capillary, $f_{c}(\mathrm{~Hz})$ & 224 & 224 & 448 \\
\hline Damping ratio of the system, $\Gamma$ & 2.0 & 0.78 & 1.6 \\
\hline
\end{tabular}

\section{Results and Discussion}

Figure 4 compares the patterns of printed drops with various pulsation frequencies for nozzle $A$ and nozzle B at the minimum pulse voltage of jetting. The two nozzles have the same diameter $d_{n}$ of $30 \mu \mathrm{m}$ and the same natural frequency $f_{c}$ of $224 \mathrm{~Hz}$, while they have different damping ratios, as shown in Tables 1 and 2. For nozzle A, shown in Figure 4a, where the estimated damping ratio is 2.0 (overdamped), ink drops are printed at the designed drop spacing of $40 \mu \mathrm{m}$ for 3, 10, $30 \mathrm{~Hz}$, and even for $1 \mathrm{kHz}$. The pulsed voltage in Figure 4 a was $150 \mathrm{~V}$. On the other hand, for nozzle B, shown in Figure $4 \mathrm{~b}$, where the damping ratio is 0.78 (underdamped) and the pulsed voltage is $300 \mathrm{~V}$, the drop spacing becomes multiples of $40 \mu \mathrm{m}$ as pulsation frequency increases.

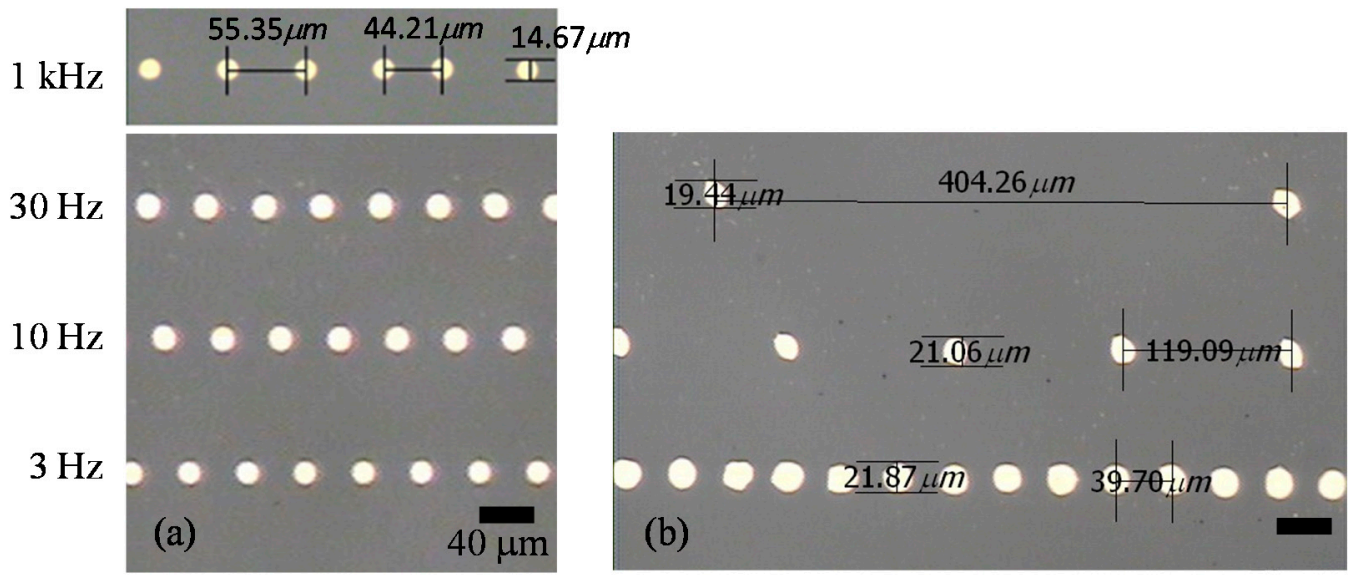

Figure 4. Printed drops of Ag ink with various pulsation frequencies of 1, 10, 30, and $1000 \mathrm{~Hz}$ for (a) overdamped system (Nozzle $\mathrm{A} ; \Gamma=2.0$ ) and (b) underdamped system (Nozzle B; $\Gamma=0.8$ ) at the minimum pulse voltages of jetting of $150 \mathrm{~V}$ and $300 \mathrm{~V}$, respectively. The two nozzles have the same diameter $d_{n}$ of $30 \mu \mathrm{m}$ and the same natural frequency $f_{c}$ of $224 \mathrm{~Hz}$, while they have different damping ratios.

Figure 5 shows the measured jetting frequency as a function of pulsation frequency. When the system is overdamped $(\Gamma>1)$, as shown for nozzle A in Figure 5, jetting frequency is in good agreement with pulsating frequency because the ink refill time is sufficiently small [15] and the meniscus stabilizes quickly after jetting. However, when system is underdamped $(\Gamma<1)$, as shown for nozzle B in Figure 5, jetting frequency does not increase with pulsation frequency but is limited to a few $\mathrm{Hz}$. This is possibly due to the capillary oscillation of the ink inside the nozzle after jetting. This result demonstrates that the overdamped EHD system is advantageous for high frequency DOD jetting. In an overdamped system, the meniscus shape and capillary force gradually change to have periodic equilibrium for a given pulsation wave. However, in an underdamped system with high frequency, there occurs instability due to an interference of capillary and pulsating waves because the capillary force varies sinusoidally in between the pulsations. 


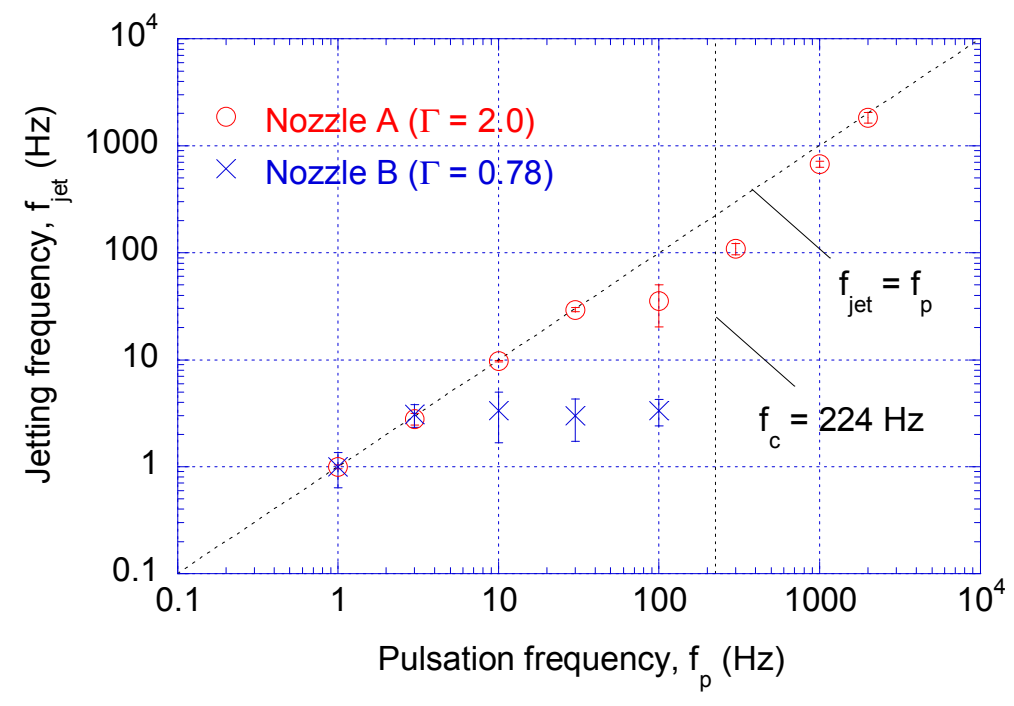

Figure 5. Measured jetting frequency as a function of pulsation frequency. Jetting frequency is in good agreement with pulsating frequency for overdamped system (Nozzle A; $\Gamma=2.0$ ), while jetting frequency does not increase with pulsation frequency at a few $\mathrm{Hz}$ for underdamped system (Nozzle B; $\Gamma=0.8)$.

Interestingly, Figure 5 also shows that, even for the overdamped nozzle A, jetting frequency deviates from the pulsation frequency near the estimated natural oscillation frequency, $f_{c}$. In order to investigate the effect of $f_{c}$ on DOD jetting, Figure 6 shows the jetting frequency as a function of the natural frequency ratio for overdamped nozzles A and C. As shown in Tables 1 and 2, the inner diameter of nozzle $\mathrm{C}$ is $15 \mu \mathrm{m}$ (one half the diameter of nozzle A) and, therefore, the estimated natural frequency is $448 \mathrm{~Hz}$ (double the natural frequency of nozzle A). The natural frequency ratios of pulsation $\alpha$ and jetting $\beta$ are the frequencies normalized by the natural frequency, respectively.

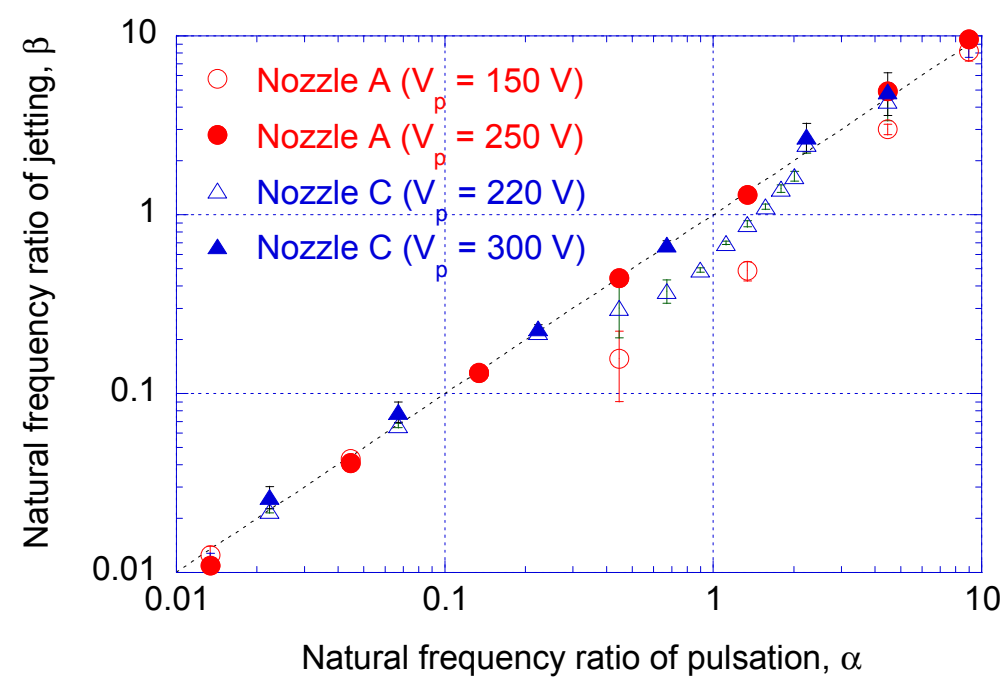

Figure 6. Natural frequency ratio of jetting as a function of natural frequency ratio of pulsation for overdamped nozzles A $\left(f_{c}=224 \mathrm{~Hz}\right)$ and $\mathrm{C}\left(f_{c}=448 \mathrm{~Hz}\right)$. Pulse voltages of $150 \mathrm{~V}$ and $220 \mathrm{~V}$ are the minimum jetting voltages for nozzles $\mathrm{A}$ and $\mathrm{C}$, respectively, while $250 \mathrm{~V}$ and $300 \mathrm{~V}$ are the increased pulse voltages of jetting for nozzles A and C, respectively.

In Figure 6, pulsed voltages $V_{p}$ of either $150 \mathrm{~V}$ for nozzle $\mathrm{A}$ or $220 \mathrm{~V}$ for nozzle $\mathrm{C}$ are close to the minimum voltage that allows DOD jetting shown in Figure 2 and Equation (1). The bias voltage $V_{b}$ 
and nozzle-substrate distance $h$ are given in Table 1. With this low pulsation voltage, the electrostatic pulsation is balanced by capillary oscillation at the nozzle tip. Therefore, when pulsation frequency approaches the natural frequency $(0.5<\alpha<2)$, the jetting frequency does not match pulsation frequency due to interference between the electrostatic pulsation and capillary oscillation. On the other hand, the effect of the resonance on distortion of the jetting frequency decreases when pulsed voltage increases to $250 \mathrm{~V}$ for nozzle A or $300 \mathrm{~V}$ for nozzle C, so that the electrostatic force becomes stronger than the capillary force.

\section{Concluding Remarks}

Characteristics of EHD jetting were experimentally investigated with various pulsating frequencies and nozzle dimensions. A simplified linear damping model was also adopted to describe the oscillating motion of Ag ink inside the nozzle. Based on the results, the effects of the damping ratio and the natural frequency were discussed.

By comparing the results of two nozzles having the same diameter and the same natural frequency, but different damping ratios, it is demonstrated that the overdamped EHD nozzle system is advantageous to support high frequency DOD jetting. When the system is overdamped, jetting frequency was in good agreement with the pulsating frequency up to $2000 \mathrm{~Hz}$. However, when the system is underdamped, the jetting frequency does not increase with the pulsation frequency, but is limited to a few $\mathrm{Hz}$, because capillary oscillation requires time to form a stabilized meniscus in an underdamped system.

It is also shown that when the pulsation frequency approaches the natural frequency $(0.5<\alpha<2)$, mismatch occurs between the jetting frequency and the pulsation frequency due to interference between the electrostatic pulsation and the capillary oscillation. This mismatch is mitigated when the pulsed voltage increases, because the electrostatic force becomes superior to the capillary force.

Acknowledgments: This work was supported by an Industrial Technology Innovation program grant no. 10052802 from Korea Evaluation Institute of Industrial Technology, funded by the Ministry of Trade, Industry and Energy of the Korean Government.

Author Contributions: S. H. Kim and J. Y. Hwang conceived and designed the experiments; S. H. Kim performed the experiments; All authors discussed the findings in paper.

Conflicts of Interest: The authors declare no conflict of interest.

\section{References}

1. Ru, C.; Luo, J.; Xie, S.; Sun, Y. A review of non-contact micro- and nano-printing technologies. J. Micromech. Microeng. 2014, 24, 053001. [CrossRef]

2. Park, J.U.; Hardy, M.; Kang, S.J.; Barton, K.; Adair, K.; Mukhopadhyay, D.K.; Lee, C.Y.; Strano, M.S.; Alleyne, A.G.; Georgiadis, J.G.; et al. High-resolution electrohydrodynamic jet printing. Nat. Mater. 2007, 6, 782-789. [CrossRef] [PubMed]

3. Li, J.L. On the meniscus deformation when the pulsed voltage is applied. J. Electrost. 2006, 64, 44-52. [CrossRef]

4. Lee, S.; Song, J.; Kim, H.; Chung, J. Time resolved imaging of electrohydrodynamic jetting on demand induced by square pulse voltage. J. Aerosol Sci. 2012, 52, 89-97. [CrossRef]

5. Li, J.; Zhang, P. Formation and droplet size of EHD drippings induced by superimposing an electric pulse to background voltage. J. Electrost. 2009, 67, 562-567. [CrossRef]

6. Jayasinghe, S.; Edirisinghe, M. Electrostatic atomization of a ceramic suspension at pico-flow rates. Appl. Phys. A 2005, 80, 399-404. [CrossRef]

7. Lee, A.; Jin, H.; Dang, H.W.; Choi, K.H.; Ahn, K.H. Optimization of experimental parameters to determine the jetting regimes in electrohydrodynamic printing. Langmuir 2013, 29, 13630-13639. [CrossRef] [PubMed]

8. Mishra, S.; Barton, K.L.; Alleyne, A.G.; Ferreira, P.M.; Rogers, J.A. High-speed and drop-on-demand printing with a pulsed electrohydrodynamic jet. J. Micromech. Microeng. 2010, 20, 095026. [CrossRef] 
9. Kwon, K.-S.; Lee, D.-Y. Investigation of pulse voltage shape effects on electrohydrodynamic jets using a vision measurement technique. J. Micromech. Microeng. 2013, 23, 065018. [CrossRef]

10. Park, J.; Kim, B.; Kim, S.-Y.; Hwang, J. Prediction of drop-on-demand (DOD) pattern size in pulse voltage-applied electrohydrodynamic (EHD) jet printing of Ag colloid ink. Appl. Phys. A 2014, 117, 2225-2234. [CrossRef]

11. Nguyen, V.D.; Byun, D. Mechanism of electrohydrodynamic printing based on ac voltage without a nozzle electrode. Appl. Phys. Lett. 2009, 94, 173509. [CrossRef]

12. Xu, L.; Wang, X.; Lei, T.; Sun, D.; Lin, L. Electrohydrodynamic deposition of polymeric droplets under low-frequency pulsation. Langmuir 2011, 27, 6541-6548. [CrossRef] [PubMed]

13. Kim, J.; Oh, H.; Kim, S.S. Electrohydrodynamic drop-on-demand patterning in pulsed cone-jet mode at various frequencies. J. Aerosol Sci. 2008, 39, 819-825. [CrossRef]

14. An, S.; Lee, M.W.; Kim, N.Y.; Lee, C.; Al-Deyab, S.S.; James, S.C.; Yoon, S.S. Effect of viscosity, electrical conductivity, and surface tension on direct-current-pulsed drop-on-demand electrohydrodynamic printing frequency. Appl. Phys. Lett. 2014, 105, 214102. [CrossRef]

15. Stachewicz, U.; Yurteri, C.U.; Marijnissen, J.C.M.; Dijksman, J.F. Stability regime of pulse frequency for single event electrospraying. Appl. Phys. Lett. 2009, 95, 224105. [CrossRef]

16. Marginean, I.; Nemes, P.; Vertes, A. Order-Chaos-Order Transitions in Electrosprays: The Electrified Dripping Faucet. Phys. Rev. Lett. 2006, 97, 064502. [CrossRef] [PubMed]

17. Eyring, C.; Mackeown, S.; Millikan, R. Fields currents from points. Phys. Rev. 1928, 31, 900. [CrossRef]

18. Relative Permittivity-The Dielectric Constant. Available online: http:/ / www.engineeringtoolbox.com/ relative-permittivity-d_1660.html (accessed on 8 May 2017.

19. Stachewicz, U.; Dijksman, J.F.; Burdinski, D.; Yurteri, C.U.; Marijnissen, J.C. Relaxation times in single event electrospraying controlled by nozzle front surface modification. Langmuir 2009, 25, 2540-2549. [CrossRef] [PubMed]

(C) 2018 by the authors. Licensee MDPI, Basel, Switzerland. This article is an open access article distributed under the terms and conditions of the Creative Commons Attribution (CC BY) license (http:/ / creativecommons.org/licenses/by/4.0/). 